\title{
Symptomkontrolle bei Herzinsuffizienzpatienten - was tun bei abfallender GFR und bei Hyperkaliämie?
}

\author{
Symptom control in heart failure patients - \\ how to handle GFR decrease and hyperkalaemia
}

\section{(c) (1) $(9)$}

Autoren

Vincent Brandenburg ${ }^{1}$, Johann Bauersachs ${ }^{2}$, Michael Böhm³ ${ }^{3}$ Danilo Fliser ${ }^{4}$, Stefan Frantz ${ }^{5}$, Norbert Frey ${ }^{6}$, Gerd Hasenfuß $ß^{7}$, Jan T. Kielstein ${ }^{8}$

\section{Institute}

1 Klinik für Kardiologie, Nephrologie und Internistische Intensivmedizin, Rhein-Maas-Klinikum, Würselen

2 Klinik für Kardiologie und Angiologie, Medizinische Hochschule Hannover

3 Innere Medizin III - Kardiologie, Angiologie und Internistische Intensivmedizin, Universitätsklinikum des Saarlandes, Homburg/Saar

4 Innere Medizin IV - Nieren- und Hochdruckkrankheiten, Universitätsklinikum des Saarlandes, Homburg/Saar

5 Medizinische Klinik und Poliklinik I (Kardiologie, Endokrinologie, Nephrologie, Pneumologie, Intensivund Notfallmedizin) Universitätsklinikum Würzburg

6 Klinik für Innere Medizin III (Schwerpunkt Kardiologie, Angiologie und Intensivmedizin), Universitätsklinikum Schleswig-Holstein, Kiel

7 Herzzentrum, Abt. Kardiologie und Pneumologie, Universitätsmedizin Göttingen

8 Klinik für Nephrologie, Blutreinigung und Rheumatologie, Klinikum Braunschweig

Schlüsselwörter

Herzinsuffizienz, Niereninsuffizienz, glomeruläre

Filtrationsrate, Hyperkaliämie

Key words

heart failure, chronic kidney disease, glomerular filtration rate, hyperkalaemia

online publiziert 22.01.2021

Bibliografie

Dtsch Med Wochenschr 2021; 146: e47-e55

DOI 10.1055/a-1307-8652

ISSN 0012-0472

(c) 2021. The Author(s).

This is an open access article published by Thieme under the terms of the Creative Commons Attribution-NonDerivative-NonCommercial License, permitting copying and reproduction so long as the original work is given appropriate credit. Contents may not be used for commecial purposes, or adapted, remixed, transformed or built upon. (https://creativecommons.org/licenses/by-nc-nd/4.0/)

Georg Thieme Verlag KG, Rüdigerstraße 14,

70469 Stuttgart, Germany
Korrespondenzadresse

Prof. Dr. Dr. Vincent Brandenburg

Klinik für Kardiologie, Nephrologie und Internistische

Intensivmedizin

Rhein-Maas-Klinikum, Mauerfeldchen 25, 52146 Würselen

vmbrandenburg@aol.com

\section{ZUSAMMENFASSUNG}

Bei Patienten mit Herzinsuffizienz und reduzierter Ejektionsfraktion wird durch eine optimierte medikamentöse Therapie sowohl die Symptomkontrolle verbessert als auch die Mortalität gesenkt. Eckpfeiler der Herzinsuffizienztherapie sind dabei Medikamente mit Einfluss auf das Renin-Angiotensin-Aldosteron-System, sogenannte RAAS-Inhibitoren. Dieser Artikel stellt einen kardiologisch-nephrologischen Konsens zur praxisorientierten Hilfestellung bei abnehmender glomerulärer Filtrationsrate oder Anstieg des Serum-Kaliumspiegels vor. Dies sind die 2 häufigsten Gründe für eine Dosisreduktion oder das Absetzen von prognoseverbessernden Medikamenten bei Herzinsuffizienzpatienten.

\section{ABSTRACT}

For heart failure patients with reduced ejection fraction, optimised medication improves symptom control and reduces mortality. Substances influencing the renin-angiotensinaldosteron-system, so-called RAAS-inhibitors, are the cornerstone of heart failure treatment. This article summarises a consensus between experts in cardiology and in nephrology on a pragmatic approach to manage a drop in glomerular filtration rate and incident hyperkalaemia - the two most common reasons for reducing or discontinuing heart failure medication. 


\section{Hintergrund}

Hausärztliche Praxen in Deutschland betreuen viele Patienten mit Herzinsuffizienz ( $\mathrm{HI})$ und eingeschränkter (reduzierter) Ejektionsfraktion (HFrEF). Diese Patienten weisen ein besonders hohes Risiko für Hospitalisierungen auf, die wiederum mit einem um das 2,5-Fache erhöhten Sterberisiko assoziiert sind [1]. Die Behandlung der HFrEF zielt vor allem darauf, Symptome bestmöglich zu kontrollieren, Exazerbationen zu verhindern und das Fortschreiten der Erkrankung zu verlangsamen [1]. Das Behandlungsteam, bestehend aus Hausarzt und Facharzt, muss daher gemeinsam mit dem Patienten eine nebenwirkungsarme Symptomkontrolle und Eindämmung der Krankheitsprogression durch eine ausreichend hohe Dosierung der prognoseverbessernden Medikation erreichen. Für HI-Patienten betrifft das vor allem die neurohormonale Regulation mit Betablockern und Inhibitoren des ReninAngiotensin-Aldosteron-Systems (RAASi), nämlich ACE-Hemmern (ACEI), Angiotensin-Rezeptorblockern (ARB), Angiotensin-Rezeptor-Neprilysin-Inhibitoren (ARNI) sowie den MineralokortikoidRezeptorantagonisten (MRA) Spironolacton und Epleneron. Sie sind die wichtigsten prognoseverbessernden Medikamente für HI-Patienten. Die Wirksamkeit einer Kombination dieser Präparate für die Symptomkontrolle und die Reduktion der Mortalität für HFrEF-Patienten ist mit der höchsten Evidenz- und Empfehlungsstufe der Europäischen Gesellschaft für Kardiologie (ESC) belegt. Daher sollten diese Präparate entsprechend der Empfehlungen in den Nationalen Versorgungsleitlinien zur HI auftitriert werden [2]. Bei HI-Patienten ist nicht der Blutdruck der Zielparameter, sondern die erreichte Medikamentendosis, mit der die bestmögliche Prognoseverbesserung erzielt werden kann. Manifeste, symptomatische Hypotonien sollten hierbei jedoch vermieden werden, ebenso wie symptomatische Bradykardien. Die Leitlinie für Patienten mit $\mathrm{HI}$ der ESC definiert solche Zieldosierungen, so z. B. für Ramipril $10 \mathrm{mg} / \mathrm{d}$, für Metoprolol $200 \mathrm{mg} / \mathrm{d}$ und für Spironolacton 50 mg/d [1] ( $\triangleright$ Tab. 1). Die Empfehlung in der Leitlinie, MRAs erst nach der Einstellung mit Betablockern und ACEI anzusetzen, ist historisch bedingt. Tatsächlich aber sollte bei Patienten mit einer Ejektionsfraktion $\leq 35 \%$ ein früher Einsatz von MRAs angestrebt werden, um Hospitalisierungen und Sterblichkeit zu verringern [1]. Insbesondere bei Patienten mit eingeschränkter Nierenfunktion (errechnete, d.h. abgeschätzte glomeruläre Filtrationsrate $(\mathrm{eGFR})<60 \mathrm{ml} / \mathrm{min} / 1,73 \mathrm{~m}^{2}$ ) ist unter dieser Therapie eine regelmäßige Überprüfung von Nierenfunktion und Kaliumspiegel notwendig. Für Diuretika ist keine Prognoseverbesserung bei HI-Patienten nachgewiesen, daher sollten sie nur zur Symptomkontrolle mit der niedrigsten effektiven Dosis eingesetzt werden. ARB werden als Ersatz bei Unverträglichkeit von ACEl empfohlen. Der Einsatz von ARNI kommt als Ersatz für ACEI/ARB ins Spiel, wenn ACEI/ARB in Kombination mit Betablockern und MRA keine ausreichende Symptomkontrolle erreichen, werden aber wohl in absehbarer Zeit auch als Erstlinientherapie empfohlen werden. Aus Sorge um eine Verschlechterung der Nierenfunktion oder ein Arrhythmie-Risiko aufgrund erhöhter Serum-Kaliumspiegel wird die medikamentöse Therapie nicht selten verringert oder abgebrochen und so Symptomkontrolle und Prognose des Patienten wieder verschlechtert [3]. Allerdings erfordert ein Abfall der (e)GFR oder ein Anstieg des Serum-Kaliums nicht immer eine
Umstellung der Medikation. Im Folgenden soll dargelegt werden, wie bei den beiden wichtigsten Ursachen für eine Dosisreduktion der medikamentösen Therapie bei HFrEF-Patienten aus gemeinsamer nephrologischer und kardiologischer Sicht vorzugehen ist nämlich bei einem Abfall der GFR und/oder bei einer Hyperkaliämie.

\section{Merke}

Bei Patienten mit Herzinsuffizienz ist der wichtigste therapeutische Zielparameter nicht der Blutdruck, sondern die erreichte Medikamentendosis mit Betablockern und Inhibitoren des Renin-Angiotensin-Aldosteron-Systems.

\section{Vorgehen bei abnehmender (e)GFR}

Die wahrscheinlich häufigste Ursache für den Abbruch einer Therapie mit RAAS-Blockern ist ein Abfall der GFR, der als medikamentenbedingte Verschlechterung der Nierenfunktion (fehl-)interpretiert wird [4]. Tatsächlich führen das Ansetzen oder die Dosissteigerung von ACEI, ARB oder ARNI initial zu einer Vasodilatation der efferenten glomerulären Arteriolen - dem erwünschten Effekt der Behandlung. Da bereits hyperton vorgeschädigte Nieren nur noch eingeschränkt in der Lage sind, den renalen Blutfluss zu regulieren, führt diese Vasodilatation dazu, dass der glomeruläre Blutfluss und damit auch die gemessene Filtrationsleistung der Nieren - die GFR - abfällt [5]. Entscheidend für die Prognose ist aber, dass damit auch der erhöhte Druck in den Glomeruli, ein zentraler Auslöser des chronischen Nierenversagens, abgesenkt wird. Deshalb ist ein Absetzen der Therapie mit RAASInhibitoren aufgrund eines GFR-Abfalls nur in bestimmten Fällen sinnvoll. Mehrere Metaanalysen haben gezeigt, dass ein Abfall der funktionellen Nierenparameter bei Initiation einer Therapie mit ACEI, ARBs oder MRAs nicht mit einer Prognoseverschlechterung einhergeht [6]. Tatsächlich scheinen die Inhibitoren des RAAS bei Patienten mit einer initial abnehmenden Nierenfunktion die Prognose sogar stärker zu verbessern als bei Patienten mit stabiler Nierenfunktion [7]. Eine Nachanalyse der SOLVD-Studie fand, dass nach Initiation von Enalapril bei Patientinnen und Patienten mit HFrEF ein Abfall der eGFR um bis zu $10 \%$ sogar mit einer signifikanten Senkung der Mortalität assoziiert war und ein Abfall um bis zu $35 \%$ mit einer signifikanten Senkung der Hospitalisierungsrate. Ein Abfall der eGFR von bis zu $40 \%$ war nicht mit einer erhöhten Mortalität verbunden [8]. Sofern die Nierenfunktion eines $\mathrm{HI}$-Patienten nicht bereits erheblich eingeschränkt ist (CKD-Stadium G4, d. h. eGFR $<30 \mathrm{ml} / \mathrm{min} / 1,73 \mathrm{~m}^{2}$ ), ist daher ein Abfall der eGFR um bis zu $30 \%$ kein Grund, die Therapie mit RAAS-Blockern kurzfristig zu verändern [5]. Grundsätzlich ist eine abnehmende Nierenfunktion vor allem dann kritisch, wenn sie mit einer Verschlechterung der allgemeinen klinischen Situation oder einem Anstieg der HI-Marker wie B-Typ-natriuretisches Peptid (BNP) einhergeht [6]. Bei einem stärkeren (>30\%) Abfall der (e)GFR unter ACEl oder ARB (nicht MRA!) sollte eine signifikante Nierenarterienstenose ausgeschlossen werden. Wichtig ist eine regelmäßige Überwachung der (e)GFR insbesondere in den Monaten nach der Einstellung mit oder Titration von Betablockern, ACEl, ARNI oder MRAs, um eingreifen zu können, falls die (e)GFR 
- Tab. 1 Evidenzbasierte Dosierungen prognoseverbessernder Medikamente aus zentralen Studien bei Patienten mit Herzinsuffizienz und eingeschränkter Ejektionsfraktion. Die Dosierungsangaben bei Mehrfachgabe beziehen sich auf die Einzeldosis [1].

\begin{tabular}{|c|c|c|}
\hline Medikation & Anfangsdosis (mg) & Zieldosis (mg) \\
\hline \multicolumn{3}{|l|}{ ACE-Hemmer } \\
\hline Captopril ${ }^{a}$ & 6,25 (3-mal täglich) & 50 (3-mal täglich) \\
\hline Enalapril & 2,5 (2-mal täglich) & 20 (2-mal täglich) \\
\hline Lisinoprilbb & 2,5-5,0 (1-mal täglich) & 20-35 (1-mal täglich) \\
\hline Ramipril & 2,5 (1-mal täglich) & 10 (1-mal täglich) \\
\hline Trandolaprila & 0,5 (1-mal täglich) & 4 (1-mal täglich) \\
\hline \multicolumn{3}{|l|}{ Betablocker } \\
\hline Bisoprolol & 1,25 (1-mal täglich) & 10 (1-mal täglich) \\
\hline Carvedilol & 3,125 (2-mal täglich) & 25 (2-mal täglich) $^{d}$ \\
\hline $\begin{array}{l}\text { Metoprololsuccinat } \\
\text { retard }\end{array}$ & 12,5-25 (1-mal täglich) & 200 (1-mal täglich) \\
\hline Nebivolok & 1,25 (1-mal täglich) & 10 (1-mal täglich) \\
\hline \multicolumn{3}{|l|}{ ARB } \\
\hline Candesartan & 4-8 (1-mal täglich) & 32 (1-mal täglich) \\
\hline Valsartan & 40 (2-mal täglich) & 160 (2-mal täglich) \\
\hline Losartan & 50 (1-mal täglich) & 150 (1-mal täglich) \\
\hline \multicolumn{3}{|l|}{ MRA } \\
\hline Eplerenon & 25 (1-mal täglich) & 50 (1-mal täglich) \\
\hline Spironolacton & 25 (1-mal täglich) & 50 (1-mal täglich) \\
\hline \multicolumn{3}{|l|}{ ARNI } \\
\hline Sacubitril/Valsartan & 49/51 (2-mal täglich) & 97/103 (2-mal täglich) \\
\hline
\end{tabular}

ACE $=$ Angiotension-Converting-Enzym; $A R B=$ Angiotensin-II-Rezeptorblocker; ARNI = Angiotensin-Rezeptor-Neprilysin-Inhibitoren; MRA = Mineralokortikoid-Rezeptorantagonisten.

a Zieldosis aus Studien bei Patienten nach Herzinfarkt.

b höhere Dosis reduziert Morbidität/Mortalität, aber optimale Dosis unklar.

d Maximaldosis bei Patienten über $85 \mathrm{~kg} 50 \mathrm{mg}$ 2-mal täglich

c Mortalitätsreduktion bei $\mathrm{HI}$ nicht belegt, Reduktion.

zu deutlich abnimmt. Es sei darauf hingewiesen, dass die eGFR als errechnete Schätzgröße für viele Patientenpopulationen nicht validiert ist. Dies sind z. B. kachektische [9], morbid adipöse oder sehr sportliche Patienten [10] oder solche nach Amputation von Extremitäten [11]. Bei diesen Patienten empfiehlt sich dann die klassische Bestimmung der Kreatinin-Clearance mittels Sammelurin, wobei auch der Grad der Proteinurie bestimmt werden sollte.

\section{Merke}

Die eGFR ist ein Schätzwert für die Nierenfunktion, und die Interpretation eines Abfalls sollte immer im Zusammenhang mit der klinischen Situation, insbesondere Medikamentenumstellungen, beurteilt werden.
- Tab. 2 Vorgehen bei Blutabnahme, um fehlerhaften SerumKaliummessungen vorzubeugen.

Blutabnahme zur Bestimmung des Serum-Kaliums

\begin{tabular}{|c|c|}
\hline Vorbereitung & $\begin{array}{l}\text { - Lithium-Heparinat-Röhrchen verwenden } \\
\text { - Nüchtern-Abnahme }\end{array}$ \\
\hline Abnahme & $\begin{array}{l}\text { - Faustschluss vermeiden } \\
\text { - Stauung vor Abnahme von Blut zur Bestimmung } \\
\text { des Kaliumwertes lösen }\end{array}$ \\
\hline Transport & - Möglichst rascher Transport ins Labor \\
\hline
\end{tabular}

\section{Definition und Diagnose der Hyperkaliämie}

Der zweite häufige Grund dafür, dass die Therapie insbesondere mit ACE-Hemmern und MRAs bei Patienten mit HI reduziert oder abgesetzt wird, ist das Auftreten einer Hyperkaliämie. Für die Beurteilung dieser Situation ist es wichtig zu berücksichtigen, dass Kalium kein Spuren-, sondern ein Mengenelement ist. In den Zellen liegen Konzentrationen von ca. 140 mmol/I Kalium vor; im Extrazellulärraum bei Gesunden hingegen nur 3,5$5,0 \mathrm{mmol} / \mathrm{I}$. Beim Untergang von Zellen, z. B. nach Traumata oder durch Tumor- oder Hämolyse, kann sich durch den Austritt intrazellulären Kaliums der Serumspiegel rasch erhöhen, insbesondere wenn der wichtigste Eliminationsweg über die Niere aufgrund von Exsikkose oder fortgeschrittener chronischer Nierenerkrankung nicht ausreichend funktioniert. Grundsätzlich ist ein einzelner erhöhter Messwert kritisch zu hinterfragen, denn Kaliummessungen sind in hohem Maße fehleranfällig: Da nur 0,4\% des körpereigenen Kaliums im Blut zirkulieren, 1,6\% im Gewebe verteilt sind und $98 \%$ in den Zellen vorliegen, beeinflusst eine verstärkte Hämolyse bei und nach der Blutabnahme den Kaliumwert erheblich. Bereits eine längere Venenstauung oder die Aufforderung an den Patienten, eine Faust zu bilden [12], eine zu warme Lagerung der Proben sowie lange Transportwege zum Labor können durch Zerfall von Blutzellen zu falsch erhöhten Werten führen. Diese Effekte können ggf. durch simultane Messung der Laktatdehydrogenase (LDH) ausgeschlossen werden. Die Fehleranfälligkeit der Kaliummessung wurde in einer Studie bestätigt, die zeigte, dass $44 \%$ der Patientinnen und Patienten mit Hyperkaliämie mindestens eine Kaliummessung im Normbereich hatten (Pseudonormokaliämie) und bei 30 \% der normokaliämischen Patienten ein zu hoher Kaliumwert bestimmt wurde (Pseudohyperkaliämie) [13]. In einer weiteren Studie lag die Rate der Pseudohyperkaliämie sogar bei 64,1\% [14]. Um Messfehler zu vermeiden, sollten daher die in $>$ Tab. 2 aufgeführten Punkte berücksichtigt werden.

Ausgeprägte Hyperkaliämien können zu kardialen Arrhythmien, Herzstillstand und -tod führen und erfordern daher eine Abklärung. Eine allgemein gültige Definition der Hyperkaliämie existiert nicht. Meist werden Werte über 5,0 mmol/l als milde Hyperkaliämie bezeichnet, Werte über $6,0 \mathrm{mmol} / \mathrm{l}$ als schwere Ausprägung. Aus den Serumwerten lässt sich allerdings nicht direkt die Dringlichkeit einer Intervention ableiten. Insbesondere Patienten mit chronischer Niereninsuffizienz entwickeln oft über längere Zeiträume erhöhte Kaliumspiegel und tolerieren dann Hyperkaliämien ohne Zeichen von Arrhythmien [3]. Bei diesen 
Patienten ist die Hyperkaliämie oft ein Nebenbefund bei Routineuntersuchungen. Von einer chronischen Hyperkaliämie ist auszugehen, wenn im Verlauf eines Jahres mehrmals Werte über $5 \mathrm{mmol} / \mathrm{l}$ gemessen werden. In einer Auswertung des dänischen Nationalregisters bei HI-Patientinnen und Patienten [15], die mindestens ein Schleifendiuretikum und einen ACE-Hemmer bzw. ARB erhielten, wiesen 2,5\% Serum-Kaliumwerte über 5,6 mmol/I auf und weitere $7,4 \%$ Werte über $5,0 \mathrm{mmol} / \mathrm{I}$. In verschiedenen anderen epidemiologische Erhebungen wiesen bis zu $36 \%$ aller Patienten [16] mit kardiovaskulären Erkrankungen eine Hyperkaliämie auf, wobei die Wahrscheinlichkeit mit abnehmender Nierenfunktion, steigender Zahl der Komorbiditäten und steigendem Alter zunimmt [3].

Eine zentrale Rolle für die Vermeidung kritischer hyperkaliämischer Episoden spielt ein engmaschiges Monitoring von Risikopatienten. Daher ist eine regelmäßige Messung der Kaliumspiegel bei diesen Patienten der erste Schritt zur Vermeidung von Hyperkaliämien. Die nationalen Versorgungsleitlinien zur chronischen HI empfehlen Laboruntersuchungen vor Therapiebeginn, 12 Wochen nach jeder Dosissteigerung, nach 3 Monaten und danach in halbjährlichen Abständen sowie bei Therapieänderung. Für Patienten, die MRA erhalten, werden 4-monatige Kontrollintervalle empfohlen [2]. Bei HI-Patienten unter MRA-Therapie wurden Inzidenzraten einer Hyperkaliämie von 6-12\% berichtet [3], allerdings wurde hier auch ein unzureichendes Monitoring der Kaliumspiegel und der (e)GFR festgestellt $[3,15]$. Bei ARNI wurde im Vergleich zu ACEI in einer retrospektiven Analyse der PARADIGM-HF-Studie ein geringeres Risiko für Hyperkaliämien berichtet [17].

\section{Merke}

Bei erstmalig stark erhöhten Serum-Kaliumspiegeln sollten zuerst potenzielle Messfehler abgeklärt werden.

\section{Vorgehen bei einer bestätigten Hyperkaliämie}

Eine Hyperkaliämie ist potenziell lebensbedrohlich und muss daher behandelt werden. Ob eine Senkung des Kaliumspiegels per se prognoseverbessernd ist, ist allerdings unklar. Es liegen widersprüchliche Daten vor. In einer Beobachtungsstudie bei Patientinnen und Patienten, die aufgrund einer akuten dekompensierten $\mathrm{HI}$ im Krankenhaus behandelt wurden, zeigte sich eine erhöhte 6-Monats-Sterblichkeit, wenn der Kaliumspiegel während des Krankenhausaufenthalts um mehr als $15 \%$ gesenkt wurde [18]. In einer anderen Population vergleichbarer Patienten war die Korrektur der Serum-Kaliumwerte in den Normalbereich nach Entlassung hingegen mit einer Prognoseverbesserung über die folgenden 5 Jahre verbunden [19]. Interventionelle, kontrollierte Studien dazu gibt es aber bisher nicht. Ein kritischer Punkt bei der Wahl des Vorgehens ist die genaue Interventionsschwelle bei hyperkaliämischen Patienten: Die Leitlinien der Europäischen Kardiologischen Gesellschaft für HI-Patienten empfehlen bei Werten über 6,0 mmol/l ein kurzzeitiges Absetzen von ACEI, ARB, ARNI oder MRAs und ein möglichst baldiges Wiederansetzen unter Kontrolle des Kaliumspiegels. Sie verweisen auch auf die Option, durch den Einsatz neuer Kaliumbinder wiederkehrenden Hyperkaliämien vorzubeugen [1].
- Tab. 3 Auswahl kaliumreicher Nahrungsmittel und Nahrungsergänzungsmittel [3, 34-36].

\begin{tabular}{|c|c|}
\hline $\begin{array}{l}\text { Nahrungsmittel mit } \\
\text { hohem Kaliumgehalt }\end{array}$ & Anmerkungen \\
\hline \multicolumn{2}{|l|}{ Hülsenfrüchte } \\
\hline $\begin{array}{l}\text { Obst: Aprikose, Banane, } \\
\text { Honigmelone }\end{array}$ & $\begin{array}{l}\text { Deutlich erhöhter Kaliumgehalt bei nahezu } \\
\text { allen Arten von Trockenobst und bei Säften } \\
\text { (z. B. Trauben/Wein, Orangen) }\end{array}$ \\
\hline $\begin{array}{l}\text { Wurzelgemüse: Kartoffel, } \\
\text { Karotte, Kohlrabi }\end{array}$ & $\begin{array}{l}\text { - gilt auch für hoch verarbeitete Kartof- } \\
\text { felprodukte wie Pommes Frites oder } \\
\text { Chips } \\
\text { - deutlich erhöhter Kaliumgehalt bei } \\
\text { Säften dieser Gemüse } \\
\text { - durch Kochen mit viel Wasser und } \\
\text { Abschütten verlieren Nahrungsmittel } \\
\text { einen erheblichen Teil ihres Kaliumge- } \\
\text { halts }\end{array}$ \\
\hline $\begin{array}{l}\text { Fruchtgemüse: Avocado, } \\
\text { Tomate, Fenchel }\end{array}$ & $\begin{array}{l}\text { - Cave: Tomatenmark, Trockenzwiebeln } \\
\text { - tiefgekühltes Gemüse auftauen und } \\
\text { Auftauwasser verwerfen, um Kalium zu } \\
\text { reduzieren }\end{array}$ \\
\hline $\begin{array}{l}\text { Blattgemüse: Grünkohl, } \\
\text { Spinat, Mangold, } \\
\text { Feldsalat }\end{array}$ & $\begin{array}{l}\text { durch gründliches Waschen kann der } \\
\text { Kaliumgehalt verringert werden }\end{array}$ \\
\hline \multicolumn{2}{|l|}{$\begin{array}{l}\text { Haselnüsse, Cashewkerne, } \\
\text { Erdnüsse, Mandeln }\end{array}$} \\
\hline Pilze & $\begin{array}{l}\text { nur Shiitake-Pilze haben einen mittleren } \\
\text { Kaliumgehalt }\end{array}$ \\
\hline $\begin{array}{l}\text { Dinkel-, Roggen-, } \\
\text { Buchweizenvollkornmehl }\end{array}$ & $\begin{array}{l}\text { zusätzlich erhöhter Kaliumgehalt, wenn } \\
\text { Pottasche (Kaliumkarbonat) als Backtrieb- } \\
\text { mittel verwendet wird (Weihnachtsge- } \\
\text { bäck) }\end{array}$ \\
\hline \multicolumn{2}{|l|}{ Weizenkleie, Weizenkeime } \\
\hline \multicolumn{2}{|l|}{$\begin{array}{l}\text { Kakao und kakaohaltige } \\
\text { Produkte }\end{array}$} \\
\hline $\begin{array}{l}\text { Alfalfa (Luzerne) -Sprossen, } \\
\text { Bambussprossen }\end{array}$ & $\begin{array}{l}\text { Alfalfa-Sprossen werden auch zur Selbst- } \\
\text { medikation genutzt }\end{array}$ \\
\hline Salzersatz & $\begin{array}{l}\text { Kaliumchlorid ist der häufigste Bestandteil } \\
\text { von Salzersatz }\end{array}$ \\
\hline enterale Ernährung & $\begin{array}{l}\text { Trinknahrung, die nicht speziell für } \\
\text { Nierenpatienten entwickelt wurde, kann } \\
\text { erhebliche Mengen Kalium enthalten }\end{array}$ \\
\hline
\end{tabular}

Aus Sicht der Autoren kann folgendes Vorgehen empfohlen werden: Wenn Patienten mit einer eGFR $>30 \mathrm{ml} / \mathrm{min} / 1,73 \mathrm{~m}^{3}$ die Kalium-Normwerte überschreiten, sollten Werte $\leq 6,0 \mathrm{mmol} / \mathrm{I}$ ohne das Vorliegen klinischer Zeichen zuerst durch eine weitere Messung überprüft werden. Bei bestätigter Hyperkaliämie sollten „Diätsünden“ als Ursache einer kurzfristigen Erhöhung des Kaliumspiegels aktiv mit dem Patienten abgeklärt werden, z. B. anhand der Liste in $\triangleright$ Tab.3. Ein weiterer potenzieller Auslöser erhöhter Kaliumspiegel sind Exsikkose und Oligurie, die die Kaliumexkretion durch die Niere blockieren. Eine ausgeprägte Exsikkose, die vom Patienten nicht innerhalb der nächsten $24 \mathrm{~h}$ oral ausgeglichen werden kann, sollte ggf. durch Kristalloid-Infusion 
- Tab.4 Eine Hyperkaliämie begünstigende und kaliumhaltige frei verkäufliche Medikamente und Medizinprodukte [3].

\begin{tabular}{|l|l|}
\hline Wirksubstanzen & \multicolumn{2}{|l|}{ Anwendungsgebiete } \\
\hline \multicolumn{2}{|l|}{ eine Hyperkaliämie begünstigend } \\
\hline NSAIDs & \multicolumn{2}{|l|}{ Schmerzmittel } \\
\hline Mannitol & Osmodiuretikum, Zuckerersatz \\
\hline kaliumhaltig & \\
\hline $\begin{array}{l}\text { Aminosäuren-Präparate: } \\
\text { Arginin, Lysin }\end{array}$ & Nahrungssupplemente \\
\hline $\begin{array}{l}\text { Heilkräuter und Zuberei- } \\
\text { tungen daraus: Löwenzahn, } \\
\text { Brennnessel, Melde, Taiga- } \\
\text { wurzel (Sibirischer Ginseng) }\end{array}$ & $\begin{array}{l}\text { eingesetzt u. a. bei Gallenleiden, } \\
\text { Leberbeschwerden, Müdigkeit, }\end{array}$ \\
\hline $\begin{array}{l}\text { Nierenleiden, Rheuma und zur } \\
\text { Stäri-Saft (Morinda, } \\
\text { Indische Maulbeere) }\end{array}$ & $\begin{array}{l}\text { - Immunstärkung, Schlafbe- } \\
\text { schwerden }\end{array}$ \\
\hline & $\begin{array}{l}\text { in Kasuistik als Ursache einer } \\
\text { Hyperkaliämie bei CKD } \\
\text { beschrieben }\end{array}$ \\
\hline
\end{tabular}

korrigiert werden. Bei Patienten mit eingeschränkter Nierenfunktion sollte eine chronische metabolische Azidose durch Blutgasanalyse abgeklärt werden. Bei Serum-Bikarbonatspiegeln $<22 \mathrm{mmol} / \mathrm{l}$ sollte dieses durch orales Bikarbonat eingestellt werden, da die Depletion der körpereigenen Säurepuffer ebenfalls den Anstieg der Kaliumspiegel begünstigt [20].

Sind kaliumreiche Lebensmittel, Exsikkose und chronische metabolische Azidose als Ursachen ausgeschlossen, sollte die Relevanz der Medikation für die Kontrolle und Prognose der $\mathrm{HI}$ überprüft werden. Dazu sollte die Einnahme frei verkäuflicher Medikamente abgefragt werden, insbesondere von nichtsteroidalen Antiphlogistika (NSAIDs) ( $\bullet$ Tab. 4). Die Einnahme von NSAIDs ist eine unterschätzte Ursache der Hyperkaliämie, wie eine gerade veröffentlichte, große kanadische Beobachtungsstudie bei Patienten über 66 Jahren belegt [21]. Auch selektive COX2-Hemmer können eine Hyperkaliämie begünstigen. Zu Paracetamol liegen hingegen keine Berichte über eine mit der Einnahme assoziierte Hyperkaliämie vor; bei Überdosierung verursacht es allerdings eine Hypokaliämie [22]. Auch subkutan appliziertes Heparin, das sich Patienten nach einem Krankenhausaufenthalt selbst spritzen, kann eine Hyperkaliämie auslösen [23]. Schließlich ist auch eine Reihe verschreibungspflichtiger Medikamente mit einer Erhöhung des Kaliumspiegels assoziiert ( $\bullet$ Tab. 5).

Patienten mit bestätigter Hyperkaliämie und symptomatischen Arrhythmien bzw. suggestiven EKG-Veränderungen müssen zur Akuttherapie sofort hospitalisiert werden. Ein zentrales diagnostisches Instrument, um die Dringlichkeit einer sofortigen Therapie bei einer bestätigten Hyperkaliämie einzuschätzen, ist das EKG. Wenn die erhöhten Kaliumspiegel die Reizleitung am Herzen beeinflussen, zeigen sich charakteristische Veränderungen in Form von Blockbildern, hohen, zeltförmigen T-Wellen und zunehmender Verbreiterung des QRS-Komplexes ( $\bullet$ Abb. 1). Bei Patienten mit Schrittmacher oder vorbestehenden Reizleitungsstörungen wie
- Tab. 5 Eine Hyperkaliämie begünstigende und kaliumhaltige verschreibungspflichtige Medikamente und Medizinprodukte [3]

\begin{tabular}{|c|c|}
\hline Wirksubstanzen & Anwendungsgebiete, Anmerkungen \\
\hline \multicolumn{2}{|c|}{ eine Hyperkaliämie begünstigend } \\
\hline $\begin{array}{l}\text { kaliumsparende Diuretika: } \\
\text { Triamteren, Amilorid }\end{array}$ & $\begin{array}{l}\text { - Hypertonie, Herzinsuffizienz } \\
\text { - Triamteren wird auch in Kombina- } \\
\text { tion mit Hydrochlorothiazid } \\
\text { eingesetzt }\end{array}$ \\
\hline Betablocker & $\begin{array}{l}\text { Hypertonie, Herzinsuffizienz; Effekt } \\
\text { eher gering im Vergleich zu anderen } \\
\text { Inhibitoren des Renin-Angiotensin- } \\
\text { Systems }\end{array}$ \\
\hline $\begin{array}{l}\text { ACE-Hemmer und } \\
\text { AT1-Blocker }\end{array}$ & Hypertonie, Herzinsuffizienz \\
\hline ARNI: Sacubitril-Valsartan & Herzinsuffizienz \\
\hline $\begin{array}{l}\text { MRAs: Spironolacton, } \\
\text { Epleneron }\end{array}$ & Herzinsuffizienz, Hypertonie \\
\hline Digitalis & $\begin{array}{l}\text { - Herzinsuffizienz, Vorhofflimmern } \\
\text { - Herzglykoside sind auch in anderen } \\
\text { Pflanzen (z. B. Maiglöckchen, } \\
\text { Oleander) und Drogen (z. B. } \\
\text { Krötengifte) enthalten }\end{array}$ \\
\hline Aliskiren & Hypertonie \\
\hline $\begin{array}{l}\text { Calcineurin-Inhibitoren: } \\
\text { Cyclosporin A, Tacrolimus }\end{array}$ & Immunsuppression \\
\hline Pentamidin & Antiprotozoikum \\
\hline $\begin{array}{l}\text { Cotrimoxazol } \\
\text { (Trimethoprim/ } \\
\text { Sulfamethoxazol) }\end{array}$ & Antibiotikum \\
\hline $\begin{array}{l}\text { Heparin (auch } \\
\text { niedermolekular) }\end{array}$ & Antikoagulation \\
\hline \multicolumn{2}{|l|}{ kaliumhaltig } \\
\hline Penicillin-G & $\begin{array}{l}\text { Antibiotikum, Applikation als } \\
\text { Kalium-Salz }\end{array}$ \\
\hline$\varepsilon$-Aminocapronsäure & Antifibrinolytikum \\
\hline Blutprodukte & $\begin{array}{l}\text { Kalium wird durch Hämolyse } \\
\text { freigesetzt }\end{array}$ \\
\hline
\end{tabular}

z. B. Linksschenkelblock kann mithilfe des EKGs allerdings nicht zuverlässig eine Beteiligung der Hyperkaliämie an der EKG-Veränderung ausgeschlossen werden. Das EKG ist auch nicht geeignet, um eine vermutete Hyperkaliämie auszuschließen [24]. Erhöhte extrazelluläre Kaliumspiegel reduzieren im ganzen Körper das intrazu extrazelluläre Konzentrationsgefälle des Kaliums, das für den Ausstrom von Kaliumionen während der Repolarisierung von Neuronen notwendig ist. Dies führt neben Herzrhythmusstörungen auch zu Muskelschwäche oder Krämpfen und Parästhesien und ist daher diagnostisch relevant bei der Identifikation einer dekompensierten Hyperkaliämie. Zeigen sich keine akuten Zeichen von Reizleitungsstörungen und ist kein weiterer kurzfristiger Anstieg des Kaliumspiegels zu erwarten, kann eine Hyperkaliämie $\leq 6,0 \mathrm{mmol} / \mathrm{I}$ ambulant behandelt bzw. kontrolliert werden. Bei bestätigten höheren Kaliumwerten sollte eine Krankenhauseinweisung erfolgen. 


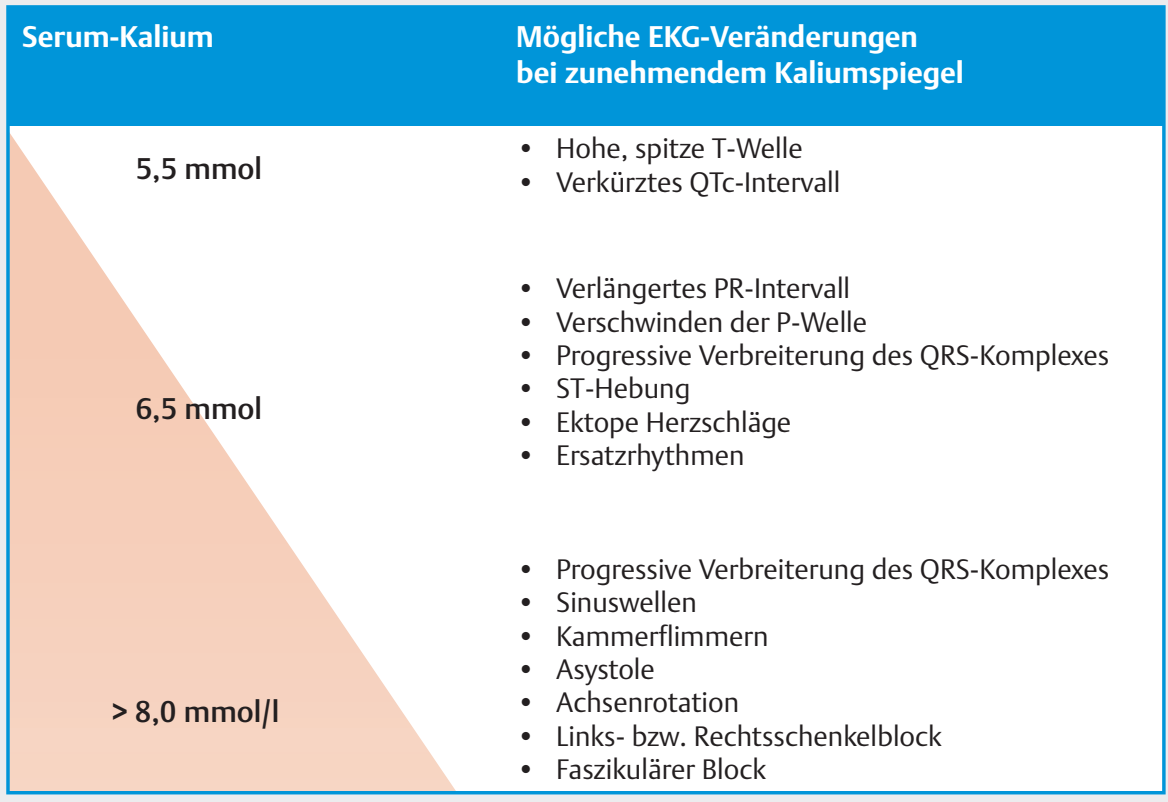

- Abb. 1 Charakteristische EKG-Veränderungen bei Hyperkaliämie [37, 38].

\section{Merke}

Bei Hyperkaliämie sollten systematisch alle potenziellen Ursachen geprüft werden, bevor die Medikation umgestellt wird. Die Dringlichkeit einer akuten Korrektur kann mittels EKG eingeschätzt werden.

\section{Dosisreduktion oder Kaliumkontrolle?}

Bleiben nach dem Ausschlussverfahren nur die prognoseverbessernden Medikamente als wahrscheinlicher Verursacher einer Hyperkaliämie bei HI-Patienten übrig, sollte sorgfältig abgewogen werden, inwieweit eine Dosisreduktion oder sogar ein Absetzen notwendig bzw. sinnvoll ist. Zudem haben sich durch die Zulassung neuer Kaliumbinder Alternativen ergeben, mit denen sich ein Abbruch der Therapie umgehen lässt. Grundsätzlich können neben MRAs und ACEl auch Betablocker eine Hyperkaliämie auslösen, daher sollte im individuellen Fall keine Präparatgruppe automatisch abgesetzt werden. Wenn irgend möglich sollte vermieden werden, eines der prognoseverbessernden Medikamente ganz abzusetzen. Dafür sprechen 3 Gründe: Aufgrund der Pharmakokinetik der Medikamente kann bei akuter Hyperkaliämie so keine rasche Stabilisierung der Kaliumwerte erreicht werden; so beträgt die Halbwertszeit für den wichtigsten aktiven Metaboliten von Spironolacton, Canrenon, 20 Stunden [25]. Zudem mutet ein Absetzen dem Patienten die mit dem Absetzen verbundenen Nebenwirkungen zu, insbesondere eine mögliche Verschlechterung der Herzfunktion [26]. Schließlich kann das Absetzen auch die Adhärenz (das Therapiebündnis) negativ beeinflussen, denn dem Patienten wird mit dem Absetzen der Eindruck vermittelt, dass er ein Medikament mit negativem Nutzen-Risiko-Profil erhalten hat [27]. Aus diesen Gründen sollte, wenn möglich, eine Dosisreduktion und kein Absetzen vorgenommen werden, solange das Serum-Kalium $<6 \mathrm{mmol} / \mathrm{l}$ liegt.
Für Patienten, bei denen der Arzt eine Dosisreduktion aufgrund der oben genannten Gründe möglichst vermeiden will, stehen 2 Optionen zur Verfügung: Kurzfristig kann mit Schleifendiuretika die Resorption von Kalium in der Niere reduziert werden. Allerdings sind sie bei Patienten mit bereits reduzierter Nierenfunktion (eGFR $<60 \mathrm{ml} / \mathrm{min} / 1,73 \mathrm{~m}^{2}$ ) nur kurzfristig geeignet, um das Serum-Kalium einzustellen. Insbesondere dann, wenn eine Reduktion der HI-Medikamente dazu führen würde, dass Symptomkontrolle und Prognoseverbesserung sich deutlich verschlechtern, können Kaliumbinder erwogen werden. Sie eigenen sich auch zur Stabilisierung von Patienten mit wiederholten akuten Hyperkaliämien. Eine Bestätigung dieses Vorgehens in langfristigen Outcome-Studien steht noch aus. Bisher waren ausschließlich Polystyrolsulfonate verfügbar, die im Darm Kalium im Austausch gegen Natrium (SPS) oder Kalzium (CPS) binden. Diese in den 60er-Jahren zugelassenen Medikamente haben eine schwache Wirkevidenz. So fand ein Cochrane-Review keine Wirkung von SPS bei akuter Hyperkaliämie [28]. Zudem sollte das Risiko für Nebenwirkungen bedacht werden: Eine 2019 in JAMA publizierte Studie fand eine Verdopplung des Risikos für gastrointestinal bedingte Hospitalisierungen bei älteren Patientinnen und Patienten ( $\geq 66$ Jahre) in den ersten 30 Tagen nach Verschreibung von SPS [29]. Die neuen Kaliumbinder Patiromer Sorbitex Calcium (PSC/Veltassa ${ }^{\circledR}$ ) oder Natrium Zirkonium Cyclosilikat (ZS-9/Lokel$\mathrm{ma}^{\circledR}$ ) haben in den Zulassungsstudien dagegen belegt, dass sie eine stabile, wo nötig auch längerfristige nebenwirkungsarme Einstellung der Kaliumspiegel ermöglichen [30-32]. Sie können daher auch bei einem erhöhten Risiko für einen Rebound der Kaliumwerte nach durchgemachter Hyperkaliämie sinnvoll sein [3]. Nach Verschreibung eines Kaliumbinders sollte innerhalb von 2-3 Tagen eine Überprüfung des Kaliumspiegels erfolgen, danach nach einer Woche und anschließend bei jeder Routinekonsulta- 


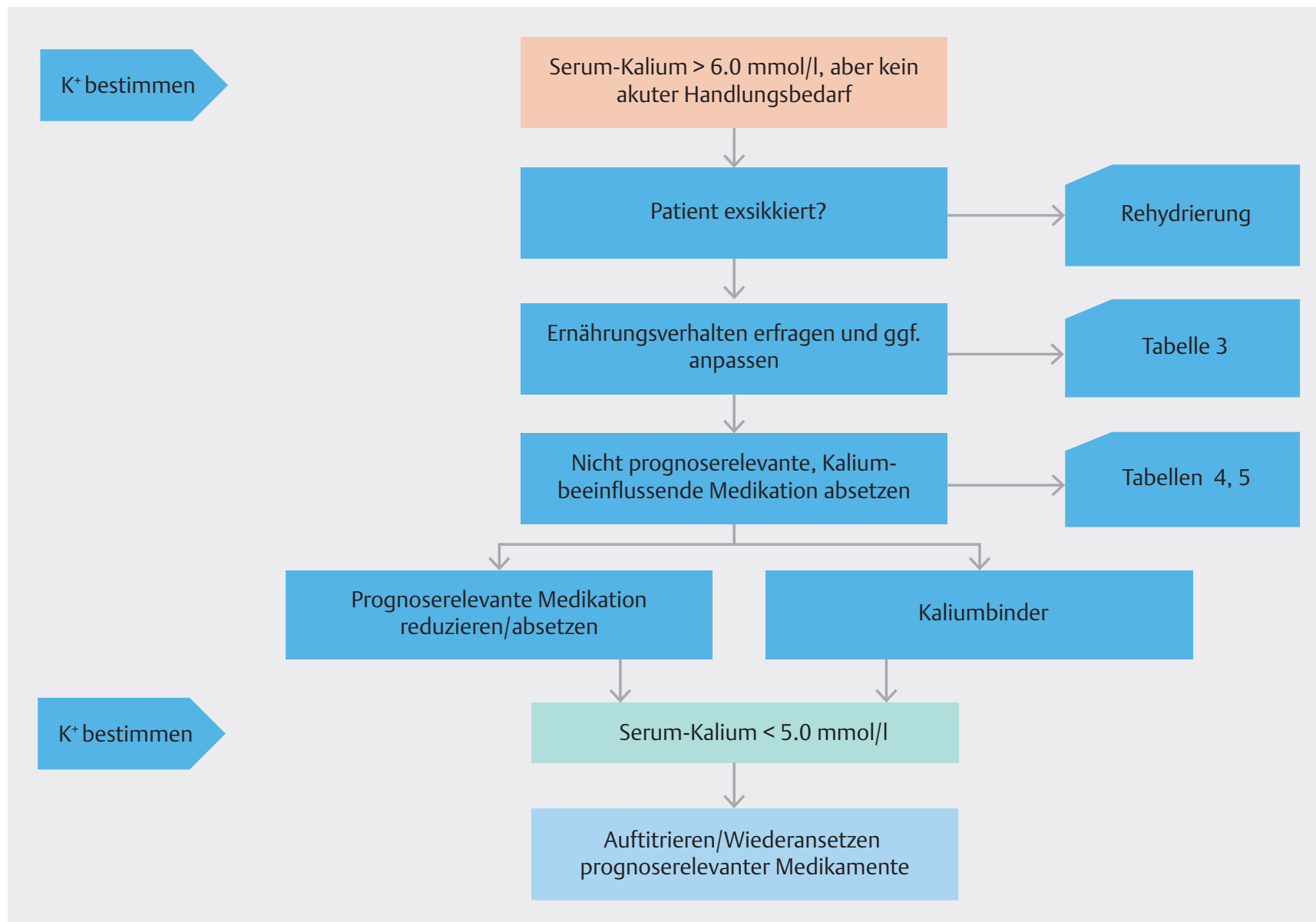

- Abb. 2 Konsensempfehlung zum möglichen Vorgehen bei HFrEF-Patienten mit nicht akut behandlungspflichtiger Hyperkaliämie. Die systematische Abklärung der Ursachen kann nach individueller Situation des Patienten angepasst werden.

tion, mindestens aber alle 6 Monate. Kalium-Zielspiegel sollten Werte $<5,0 \mathrm{mmol} / \mathrm{l}$ sein, um einen Sicherheitspuffer bei kurzfristigen akuten Anstiegen sicherzustellen. Aktuell sind in Deutschland nur Polystyrolsulfonate (SPS, CPS) und Patiromer verfügbar.

Unabhängig davon, wie die Hyperkaliämie unter Kontrolle gebracht worden ist, sollte bei Patienten mit stabilem Blutdruck und stabiler Nierenfunktion bei Kaliumwerten $<5,0 \mathrm{mmol} / \mathrm{I}$ zumindest ein Versuch gemacht werden, ein herabdosiertes oder sogar abgesetztes prognoseverbesserndes Medikament wieder leitliniengerecht einzusetzen. Die Relevanz dieser Maßnahme kann dem Patienten durch den Hinweis vermittelt werden, dass die Präparate die Herzfunktion verbessern und das Mortalitätsrisiko senken. Bei Patienten unter aktiver Kontrolle der Hyperkaliämie durch Kaliumbinder ist eine Überwachung des Kaliumspiegels bei jeder Vorstellung des Patienten besonders wichtig, auch um das Risiko einer Hypokaliämie zu minimieren. Für eine langfristig erfolgreiche Minimierung des Nebenwirkungsrisikos einer Hyperkaliämie ist ein regelmäßiges Monitoring von größter Bedeutung [16, 33]. Eine Übersicht des vorgeschlagenen Vorgehens findet sich in

\section{- Abb. 2.}

\section{Fazit}

Die medikamentöse Therapie mit Betablockern, ACE-Hemmern, ARBs und ARNIs sowie MRAs bei HFrEF-Patienten sollte nur nach Prüfung aller alternativen Ursachen und Optionen bei GFR-Abfall oder Auftreten von Hyperkaliämien herabtitriert oder gar abgesetzt werden. Bei Patienten mit Hyperkaliämie eröffnen neue Kaliumbinder eine ergänzende Möglichkeit, wiederkehrende Hyperkaliämien langfristig zu kontrollieren und eine stabile prognoserelevante optimierte Therapie der HI zu unterstützen ( $\triangleright$ Abb. 2).

\section{KERNAUSSAGEN}

- Bei Herzinsuffizienzpatienten mit einer eGFR > $30 \mathrm{ml} / \mathrm{min} /$ $1,73 \mathrm{~m}^{2}$ ist eine Abnahme der eGFR um bis zu $30 \%$ nach Ansetzen oder Auftitrieren der Therapie mit RAAS-Inhibitoren kein Grund, die Medikamente wieder abzusetzen.

- Vor dem Absetzen prognoseverbessernder Medikamente aufgrund einer Hyperkaliämie sollten Ernährung, Exsikkose, chronische metabolische Azidose und andere Medikamente mit Einfluss auf den Kaliumhaushalt als Ursache ausgeschlossen werden. 
- Zeigen sich keine akuten Zeichen von Reizleitungsstörungen, kann eine Hyperkaliämie bis $6,0 \mathrm{mmol} / \mathrm{l}$ ambulant behandelt bzw. kontrolliert werden.

- Sind prognoserelevante Medikamente die wahrscheinlichste Ursache einer Hyperkaliämie, sollte möglichst deren Dosis reduziert werden, anstatt sie abzusetzen.

- Für eine langfristig erfolgreiche Minimierung des Nebenwirkungsrisikos ist ein regelmäßiges Monitoring des Serum-Kaliumspiegels von größter Bedeutung.

\section{Interessenkonflikte}

Die Grundzüge dieses Reviews wurden durch die Autoren bei einem Expertentreffen in Frankfurt entworfen. Die Ausarbeitung des Manuskripts und die Abstimmung der verschiedenen Fassungen mit den Autoren erfolgte durch Dr. rer. nat. Christoph Messer, DBM Wissen schafft GmbH. Das Expertentreffen und die redaktionelle Unterstützung wurden von Vifor Pharma Deutschland finanziert.

Die Autoren benennen folgende potenzielle Interessenkonflikte:

B: Honorare für Vortrags- und Beratungstätigkeit: Bayer, BMS, Boehringer Ingelheim, CVRx, Daichii Sankyo, MSD, Novartis, Pfizer, Servier, Vifor, AstraZeneca, Abiomed, Abbott, Medtronic sowie Forschungsunterstützung von Zoll, CVRx, Vifor, Abiomed

MB: Stipendien der DFG, Unterstützung durch Abbott, Amgen, AstraZeneca, Bayer, Boehringer Ingelheim, Medtronic, Novartis, Servier, Vifor VB: Vortragshonorare Vifor Pharma

DF: Unterstützung durch Astra-Zeneca, Vifor Pharma

NF: Beratungshonorare Vifor Pharma

SF: Studien/Honorare/Advisory Boards AMGEN Europe, AstraZeneca,

Bayer Vital, Boehringer Ingelheim, Bristol-Meyers Squibb GmbH, Daiichi Sankyo, MSD, Novartis, Pfizer, Sanofi, Servier, Vifor GH: Honorare für Vorträge: Berlin Chemie; Beratung: Corvia; Vorträge und Beratung: Novartis, Servier, Vifor Pharma; Vorträge, Beratung und Studien: Impulse Dynamics; Editor: Springer JTK: Vortragshonorare Vifor Pharma

Literatur

[1] Ponikowski P, Voors AA, Anker SD et al. 2016 ESC Guidelines for the diagnosis and treatment of acute and chronic heart failure: The Task Force for the diagnosis and treatment of acute and chronic heart failure of the European Society of Cardiology (ESC)Developed with the special contribution of the Heart Failure Association (HFA) of the ESC. Eur Heart J 2016; 37 (27): 2129-2200. doi:10.1093/eurheartj/ehw128

[2] Bundesärztekammer, Kassenärztliche Bundesvereinigung (KBV), Arbeitsgemeinschaft der Wissenschaftlichen Medizinischen Fachgesellschaften (AWMF). Nationale VersorgungsLeitlinie Chronische Herzinsuffizienz [Langfassung]. Im Internet (Stand: 19.12.2019): https://www.leitlinien.de/ mdb/downloads/nvl/herzinsuffizienz/herzinsuffizienz-3aufl-vers1-lang.pdf

[3] Rosano GMC, Tamargo J, Kjeldsen KP et al. Expert consensus document on the management of hyperkalaemia in patients with cardiovascular disease treated with renin angiotensin aldosterone system inhibitors: coordinated by the Working Group on Cardiovascular Pharmacotherapy of the European Society of Cardiology. Eur Heart ] Cardiovasc Pharmacother 2018; 4 (3): 180-188. doi:10.1093/ehjcvp/pvy015

[4] Maeder MT, Rickli H, Pfisterer ME et al. Incidence, clinical predictors, and prognostic impact of worsening renal function in elderly patients with chronic heart failure on intensive medical therapy. Am Heart J 2012; 163 (3): 407-414, 414.e1. doi:10.1016/j.ahj.2011.12.003
[5] Clark AL, Kalra PR, Petrie MC et al. Change in renal function associated with drug treatment in heart failure: national guidance. Heart 2019; 105 (12): 904-910. doi:10.1136/heartjnl-2018-314158

[6] Damman K, Testani JM. The kidney in heart failure: an update. Eur Heart J 2015; 36 (23): 1437-1444. doi:10.1093/eurheartj/ehv010

[7] Clark H, Krum H, Hopper I. Worsening renal function during reninangiotensin-aldosterone system inhibitor initiation and long-term outcomes in patients with left ventricular systolic dysfunction. Eur J Heart Fail 2014; 16 (1): 41-48. doi:10.1002/ejhf.13

[8] McCallum W, Tighiouart $\mathrm{H}$, Ku E et al. Acute declines in estimated glomerular filtration rate on enalapril and mortality and cardiovascular outcomes in patients with heart failure with reduced ejection fraction. Kidney Int 2019. doi:10.1016/j.kint.2019.05.019

[9] Zhou Y, Hellberg M, Svensson P et al. Sarcopenia and relationships between muscle mass, measured glomerular filtration rate and physical function in patients with chronic kidney disease stages $3-5$. Nephrol Dial Transplant 2018; 33 (2): 342-348. doi:10.1093/ndt/gfw466

[10] Milic R, Banfi G, Del Fabbro M et al. Serum creatinine concentrations in male and female elite swimmers. Correlation with body mass index and evaluation of estimated glomerular filtration rate. Clin Chem Lab Med 2011; 49 (2): 285-289. doi:10.1515/CCLM.2011.057

[11] Aakjaer M, Houlind MB, Treldal C et al. Differences in Kidney Function Estimates Based on Creatinine and/or Cystatin C in Non-Traumatic Amputation Patients and Their Impact on Drug Prescribing. J Clin Med 2019; 8 (1). doi:10.3390/jcm8010089

[12] Lima-Oliveira G, Guidi GC, Salvagno GL et al. Estimation of the imprecision on clinical chemistry testing due to fist clenching and maintenance during venipuncture. Clin Biochem 2016; 49 (18): 1364-1367. doi:10.1016/j.clinbiochem.2016.07.007

[13] Friedman PA, Scott CG, Bailey K et al. Errors of Classification With Potassium Blood Testing: The Variability and Repeatability of Critical Clinical Tests. Mayo Clin Proc 2018; 93 (5): 566-572. doi:10.1016/ j.mayocp.2018.03.013

[14] Lee TS, Kim J, Uh Y et al. Correction Equation for Estimation of Actual Potassium Concentration in Hemolyzed Specimen. Clin Lab 2017; 63 (2): 271-275. doi:10.7754/Clin.Lab.2016.160733

[15] Aldahl M, Jensen AC, Davidsen L et al. Associations of serum potassium levels with mortality in chronic heart failure patients. Eur Heart ] 2017; 38 (38): 2890-2896. doi:10.1093/eurheartj/ehx460

[16] Bundesärztekammer, Kassenärztliche Bundesvereinigung (KBV), Arbeitsgemeinschaft der Wissenschaftlichen Medizinischen Fachgesellschaften (AWMF). Nationale VersorgungsLeitlinie Chronische Herzinsuffizienz, 2. Auflage. Version 3

[17] Desai AS, Vardeny O, Claggett B et al. Reduced Risk of Hyperkalemia During Treatment of Heart Failure With Mineralocorticoid Receptor Antagonists by Use of Sacubitril/Valsartan Compared With Enalapril: A Secondary Analysis of the PARADIGM-HF Trial. JAMA Cardiol 2017; 2 (1): 79-85. doi:10.1001/jamacardio.2016.4733

[18] Salah K, Pinto YM, Eurlings LW et al. Serum potassium decline during hospitalization for acute decompensated heart failure is a predictor of 6-month mortality, independent of $\mathrm{N}$-terminal pro-B-type natriuretic peptide levels: An individual patient data analysis. Am Heart ] 2015; 170 (3): 531-542.e1. doi:10.1016/j.ahj.2015.06.003

[19] Núñez J, Bayés-Genís A, Zannad F et al. Long-Term Potassium Monitoring and Dynamics in Heart Failure and Risk of Mortality. Circulation 2018; 137 (13): 1320-1330. doi:10.1161/CIRCULATIONAHA.117.030576

[20] Lee Hamm L, Hering-Smith KS, Nakhoul NL. Acid-base and potassium homeostasis. Semin Nephrol 2013; 33 (3): 257-264. doi:10.1016 j.semnephrol.2013.04.006

[21] Nash DM, Markle-Reid M, Brimble KS et al. Nonsteroidal anti-inflammatory drug use and risk of acute kidney injury and hyperkalemia in older adults: a population-based study. Nephrol Dial Transplant 2019; 34 (7): 1145-1154. doi:10.1093/ndt/gfz062 
[22] Waring WS, Stephen AFL, Malkowska AM et al. Acute acetaminophen overdose is associated with dose-dependent hypokalaemia: a prospective study of 331 patients. Basic Clin Pharmacol Toxicol 2008; 102 (3): 325328. doi:10.1111/j.1742-7843.2007.00176.x

[23] Torres $\mathrm{OH}$, Hernandez N, Francia E et al. Effect of prophylactic treatment with low-molecular-weight heparin bemiparin sodium on serum potassium levels: a prospective observational study. Drugs Aging 2010; 27 (5): 399-406. doi:10.2165/11535440-000000000-00000

[24] Montford JR, Linas S. How Dangerous Is Hyperkalemia? JASN 2017; 28 (11): 3155-3165. doi:10.1681/ASN.2016121344

[25] Heumann Pharma. Fachinformation Spironolacton. 2016

[26] Halliday BP, Wassall R, Lota AS et al. Withdrawal of pharmacological treatment for heart failure in patients with recovered dilated cardiomyopathy (TRED-HF): an open-label, pilot, randomised trial. Lancet 2019; 393: 61-73. doi:10.1016/S0140-6736(18)32484-X

[27] Tomson C, Tomlinson LA. Stopping RAS Inhibitors to Minimize AKI. CJASN 2019; 14 (4): 617-619. doi:10.2215/CJN.14021118

[28] Mahoney BA, Smith WA, Lo D et al. Emergency interventions for hyperkalaemia. Cochrane Database of Systematic Reviews 2005. doi:10.1002/14651858.CD003235.pub2

[29] Noel JA, Bota SE, Petrcich W et al. Risk of Hospitalization for Serious Adverse Gastrointestinal Events Associated With Sodium Polystyrene Sulfonate Use in Patients of Advanced AgeSerious Adverse Gastrointestinal Events Associated With Sodium Polystyrene Sulfonate UseSerious Adverse Gastrointestinal Events Associated With Sodium Polystyrene Sulfonate Use. JAMA Intern Med 2019; 179 (8): 1025-1033. doi:10.1001/jamainternmed.2019.0631
[30] Pitt B, Anker SD, Bushinsky DA et al. Evaluation of the efficacy and safety of RLY5016, a polymeric potassium binder, in a double-blind, placebocontrolled study in patients with chronic heart failure (the PEARL-HF) trial. Eur Heart J 2011; 32 (7): 820-828. doi:10.1093/eurheartj/ehq502

[31] Weir MR, Bakris GL, Bushinsky DA et al. Patiromer in patients with kidney disease and hyperkalemia receiving RAAS inhibitors. NEJM 2015; 372 (3): 211-221. doi:10.1056/NEJMoa1410853

[32] Packham DK, Rasmussen HS, Lavin PT et al. Sodium Zirconium Cyclosilicate in Hyperkalemia. N Engl J Med 2014; 372 (3): 222-231. doi:10.1056/NEJMoa1411487

[33] Cooper LB, Hammill BG, Peterson ED et al. Characterization of Mineralocorticoid Receptor Antagonist Therapy Initiation in High-Risk Patients With Heart Failure. Circ Cardiovasc Qual Outcomes 2017; 10: doi:10.1161/CIRCOUTCOMES.116.002946

[34] Biesalski HK, Grimm P, Nowitzki-Grimm S. Taschenatlas Ernährung. Thieme Verlag. 2015; 6. Aufl.

[35] Deutsche Gesellschaft für Ernährung e. V. Ausgewählte Fragen und Antworten zu Kalium. Im Internet (Stand: 11/2019): https://www.dge.de/ wissenschaft/weitere-publikationen/faqs/kalium/

[36] Börsteken B. Kalium-Tabelle für pflanzliche Lebensmittel. Im Internet (Stand: 11/2019): https://www.roche.de/pharma/nephrologie/ ernaehrung/kaliumtabelle.html

[37] McCullough PA, Beaver TM, Bennett-Guerrero E et al. Acute and Chronic Cardiovascular Effects of Hyperkalemia: New Insights Into Prevention and Clinical Management. Rev Cardiovasc Med 2014; 15: 11-23. doi:10.3909/ricm0727

[38] Zieschang S. Hyperkaliämie im Praxisalltag. Arzneiverordnung in der Praxis 2018; 46 (1): 59-64 